\title{
Screening the Hipparcos-based astrometric orbits of sub-stellar objects $^{\star}$
}

\author{
D. Pourbaix ${ }^{\star}, 1,2$ and F. Arenou ${ }^{3}$ \\ 1 Institut d'Astronomie et d'Astrophysique, Université Libre de Bruxelles, CP 226, Boulevard du Triomphe, 1050 \\ Bruxelles, Belgium \\ 2 Department of Astrophysical Sciences, Princeton University, Princeton NJ 08544-1001, USA \\ 3 UMR 8633 CNRS/DASGAL, Observatoire de Paris, 5 place J. Janssen, 92195 Meudon Cedex, France
}

Received 14 March 2001 / Accepted 23 April 2001

\begin{abstract}
The combination of Hipparcos astrometric data with the spectroscopic data of putative extrasolar planets seems to indicate that a significant fraction of these low-mass companions could be brown or M dwarfs (Han et al. 2001). We show that this is due to the adopted reduction procedure, and consequently that the Hipparcos data do not reject the planetary mass hypothesis in all but one cases. Additional companions, undetected so far, might also explain the large astrometric residuals of some of these stars.
\end{abstract}

Key words. methods: data analysis - astrometry - stars: planetary systems

\section{Introduction}

The first attempt to combine the spectroscopic information concerning extrasolar planetary candidates with the Hipparcos data has been done by Perryman et al. (1996). The goal was to derive upper mass limits while the radial velocity analysis gives the lower limit.

Han et al. (2001) lately investigated the mass of 30 extrasolar companions with period longer than 10 days using the Hipparcos (ESA 1997) data. For 27 systems, the inclination they derive is smaller than $20^{\circ}$ (in 8 cases, it is even less than $1^{\circ}$ ), and they conclude that half of their sample stars might have brown or M dwarf secondary.

In this paper, we first derive similar inclinations for an extended set of systems with no limitation based on the period. Such a large percentage of small inclinations is very unlikely (Sect. 2.1). Instead of explaining it with an observational bias of radial velocity surveys (Han et al. 2001), we show that the fitting procedure is responsible for the bias. Another model is then used to fit the observations. The comparison between the two sets of solutions allows us to conclude that the data seldom contain enough information to derive a reliable value of the inclination and of the semi-major axis. Such a poor reliability will be illustrated in Sect. 4.1.

Send offprint requests to: D. Pourbaix, e-mail: pourbaix@astro.ulb.ac.be

* Based on observations from the Hipparcos astrometric satellite operated by the European Space Agency (ESA 1997).

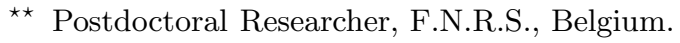

Although we show in Sect. 2.2 that the derived inclinations are strongly doubtful, we expect a very small number of small inclinations in the putative extrasolar planets sample, and the problem is therefore to distinguish between a true astrometric motion and the effect of random noise. Statistical tests are then proposed in Sect. 3 in order to detect the real massive secondaries, not due to the effect of random noise or to the perturbation of other companions.

\section{Statistical properties of the low-mass companions sample}

\subsection{The inclinations}

The method adopted for fitting the astrometric data (Mazeh et al. 1999; Halbwachs et al. 2000b; Han et al. 2001; Zucker \& Mazeh 2000) generally consists in fixing $K_{1}$ (reckoned in $\mathrm{m} / \mathrm{s}$ ) and the orbital parameters $\omega_{1}, e$, $P$ (reckoned in days), and $T$ to their spectroscopic values and to impose

$a_{\mathrm{a}} \sin i=9.19 \times 10^{-8} K_{1} P \sqrt{1-e^{2}} \varpi$.

The parallax, $\varpi$, reckoned in mas is adopted from Hipparcos and $a_{\mathrm{a}}$ is the semi-major axis obtained by astrometry. The $\chi^{2}$ thus becomes a 7 -parameter expression which is then minimised. From now on, $i_{\mathrm{C}}$ will refer to the inclination derived using that procedure.

If the orbital planes of extrasolar companions are randomly oriented, $\operatorname{Pr}(\sin i<x)=1-\sqrt{1-x^{2}}$. However, 


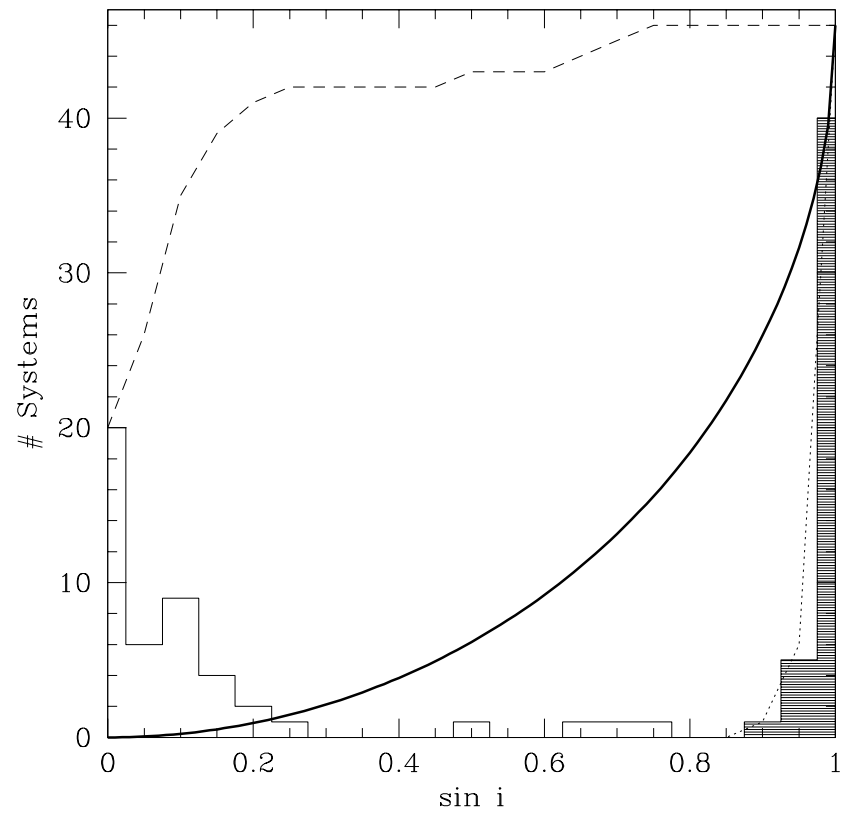

Fig. 1. Distribution of $\sin i_{\mathrm{C}}\left(\sin i_{\mathrm{T}}\right)$ plotted as open (filled) histograms. The dashed and dotted lines represent the experimental cumulative frequencies of $i_{\mathrm{C}}$ (Campbell) and $i_{\mathrm{T}}$ (Thiele-Innes) respectively. The thick continuous-line gives the theoretical cumulative frequency.

when the Hipparcos data of 46 systems $^{1}$ (Table 1 ) are fitted using the above algorithm, the distribution of $i_{\mathrm{C}}$ peaks close to 0 (Fig. 1, open histogram). In order to explain such a distribution, one can either argue that (a) the orbital planes of any unbiased sample are not randomly oriented; (b) there is a selection effect in the sample detected by radial velocity, pushing the inclinations towards small values, since the binaries have been excluded; (c) the inclinations thus derived are plain wrong. We will no longer consider explanation (a): even if there could be a preferential inclination (e.g. in clusters), that it could be 0 (i.e. towards our line of sight) would rather be anthropocentric.

Figure 1 shows that the number of systems with small inclinations $\left(i_{\mathrm{C}}\right)$ is way too large with respect to the theoretical distribution. For instance, there is only a $0.5 \%$ probability of getting a system with $\sin i<0.1$ whereas 32 systems over 46 are characterised by $\sin i_{\mathrm{C}}<0.1$.

Could a selection effect of the radial velocity surveys explain this result, as suggested by Han et al. (2001)? To show that the answer is in the negative we may take account of all companions to solar-type primaries discovered through radial velocity studies and compute the probability of getting small inclinations. Counting dwarf stars later than F5 from the spectroscopic binaries after Batten et al. (1989) and Duquennoy \& Mayor (1991) and adding the recent low-mass companions gives about 450 stars, the exact number does not matter much as will be seen below. On the average, we would expect 2 systems with $\sin i<0.1$ in

\footnotetext{
1 Based on the Catalog of extra-solar planets maintained by J. Schneider (http://www.obspm.fr/encycl/catalog.html), content in early December 2000.
}

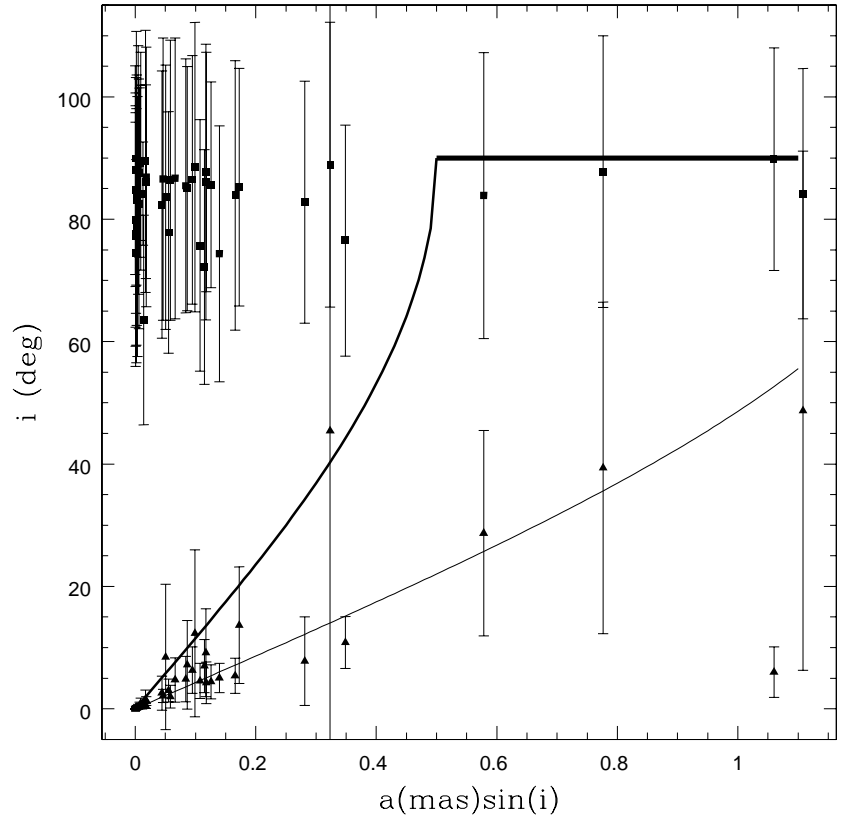

Fig. 2. Inclinations versus $a_{\mathrm{a}} \sin i$ (Eq. (1)). The triangles and squares denote $i_{\mathrm{C}}$ and $i_{\mathrm{T}}$ respectively. The thick line gives the upper bound on $i_{\mathrm{C}}$ based on $a_{\mathrm{a}} \sin i$, assuming a semi-major axis of 0.5 mas. The thin line is a least-square fit to the $i_{\mathrm{C}}$ observed values.

our sample, so that the probability to get 32 such objects is about $10^{-25}$. Even if we allowed for an underestimation by a factor 10 (!) of the inclination, to get 16 systems with $\sin (10 i)<0.1$ would have a $10^{-9}$ probability.

There may be unpublished spectroscopic companions, so let us consider all stars which can be observed. From the Tycho Catalogue, there are 72162 stars with $V<9$ and $B-V$ between 0.4 and 1.4, corresponding to F5-K8, and only a small fraction of this population has been observed indeed. Assuming a 11\% frequency of short period $(P<1000$ days) spectroscopic binaries (Halbwachs et al. 2000a), one can expect on the average 0.4 system with $\sin i<0.01$ in this whole population whereas there are 16 systems in our sample (resp. 8 in the subsample of Han et al. 2001) i.e. a $10^{-6}$ (resp. $10^{-2}$ ) probability. Facing all these unrealistic probabilities, it is clear that the hypothesis (b) above is completely ruled out.

Arenou \& Palasi (2000) and Pourbaix (2001) favoured the explanation (c): the inclinations and their formal errors may be plain wrong. The right-hand side of Eq. (1) depends on the (known) spectroscopic elements and the parallax only: as shown by Halbwachs et al. (2000b), $a_{\text {a }}$ is of the order of the astrometric precision (about 0.5 mas for the considered stars). $\sin i_{\mathrm{C}}$ is then constrained by the spectroscopic $a_{1} \sin i$ and the parallax: the smaller they are, the smaller the inclination. So, the inclinations thus obtained could have no physical meaning and could just be artifacts of the fitting procedure. 


\subsection{A test of the inclination}

In order to show that these $i_{\mathrm{C}}$ are wrong, let us relax some constraints on the orbital parameters, namely $\omega_{1}$ and $K_{1}$. The orbital parameters $e, P$, and $T$ are set to the spectroscopic values whereas the others (advantageously replaced by the Thiele-Innes constants $A, B, F$, and $G$ ) are derived from the observations. One advantage of this procedure is that, once $e, P$, and $T$ are fixed, the least-square system of equations is linear with respect to the parameters and needs no iteration to converge. Finally the parameters $\omega_{1}, a_{\mathrm{a}}, i$ and $\Omega$ are computed from the Thiele-Innes constants. We will denote by $i_{\mathrm{T}}$ the inclinations thus obtained (squares in Fig. 2). If the observations do contain the astrometric signature of the planet, the two approaches, i.e. Campbell's and Thiele-Innes' ones, both mathematically correct, should yield consistent results. We recall here that what we quote "Campbell's solution" is the determination of the Campbell parameters with the supplementary constraint of $a_{1} \sin i$ and $\omega_{1}$ to their spectroscopic value. Without this constraint the two quoted approaches are identical.

The uncertainties of $i_{\mathrm{T}}$ were derived using MonteCarlo simulation. Instead of comparing each pairs of inclinations, we compare the weighted means of the two sets (weight $=\sigma_{i}^{-2}$, and using $i$ in the first quadrant). For the Thiele-Innes and Campbell sets we obtain respectively $i_{\mathrm{T}}^{-}=83^{\circ} \pm 2.7^{\circ}$ and $\bar{i}_{\mathrm{C}}^{-}=0.02^{\circ} \pm 0.004^{\circ}(6.6 \pm 2$ for an unweighted mean). The two means are clearly discrepant.

The distribution of $i_{\mathrm{T}}$ is also given in Fig. 1. Although it looks closer to the theoretical distribution than $i_{\mathrm{C}}$ does, one can nevertheless notice an excess of edge-on orbits and a deficit of intermediate inclinations. The probability of getting no inclination below $60^{\circ}$ in this sample is $10^{-10}$. These inclinations are as unlikely as those obtained with the Campbell approach. Unfortunately, such a bias towards $90^{\circ}$ could also be consistent with a selection effect due to the spectroscopic investigations.

Actually, random noise also yields edge-on ThieleInnes orbits. Although, regardless of $e, P$ and $T$, fitting such a null signal leads to Thiele-Innes' elements normally distributed around 0 (unbiased), $i_{\mathrm{T}}(A, B, F, G)$, given by (Heintz 1978)

$\tan ^{2}\left(\frac{i_{\mathrm{T}}}{2}\right)=\sqrt{\frac{(G-A)^{2}+(B+F)^{2}}{(A+G)^{2}+(B-F)^{2}}}$,

follows a distribution centred in $\pi / 2$. When the standard deviations of the Thiele-Innes elements are all the same, $\sigma_{i} \approx 11.7^{\circ}$.

However, even a likely $i_{\mathrm{T}}$ would not mean the whole orbital solution is sensible. Indeed, even if Campbell's elements are replaced with the Thiele-Innes ones, $a_{\mathrm{a}}$ still remains closely related to the residuals of the coordinates of the star, the reason being that the computed semi-major axis roughly follows a law proportional to a $\chi(4)$ law, since $a_{\mathrm{a}} \propto \sqrt{A^{2}+B^{2}+F^{2}+G^{2}}$ (from Eq. (6)).

If there was some orbital information in the Hipparcos data, one would expect a one to one relation between the

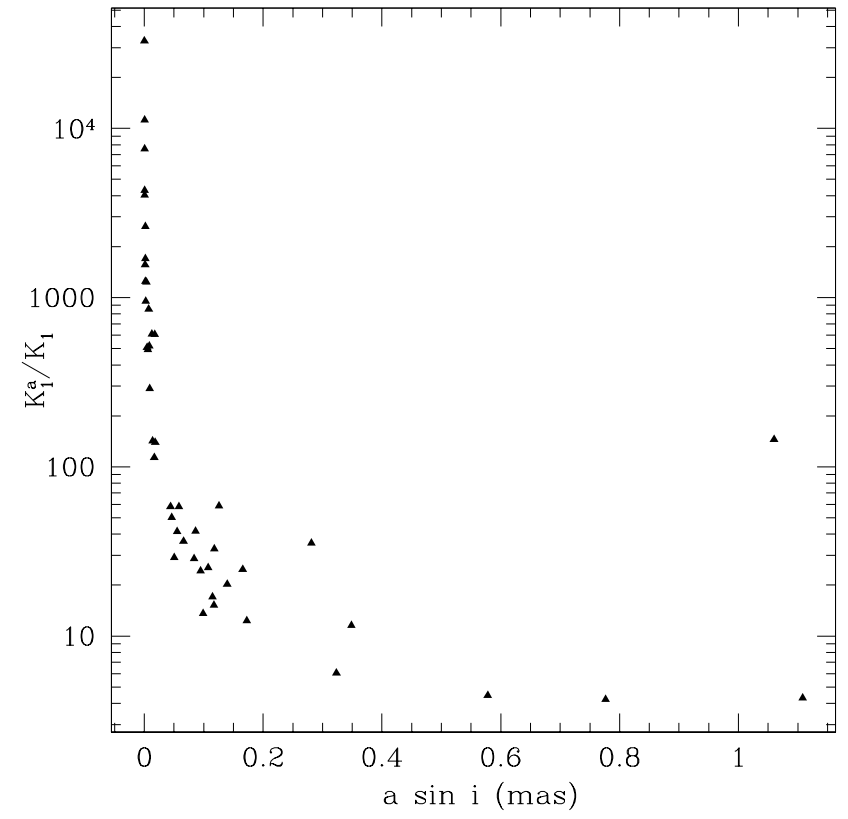

Fig. 3. Ratio of the $K_{1}$ estimate based on the Thiele-Innes elements to the spectroscopic $K_{1}$.

astrometric $K_{1 \mathrm{~T}}$ (computed using Eq. (1)) and the spectroscopic $K_{1}$ (Fig. 3). However the mean weighted difference between the two is $458 \pm 62 \mathrm{~m} / \mathrm{s}$. What happens here is that, with $a_{\mathrm{a}}$ about 0.5 mas and an inclination $i_{\mathrm{T}}$ close to $90^{\circ}$ the astrometric estimate of $K_{1}$ is almost the inverse of the spectroscopic $a_{1} \sin i \times \varpi$. Hence, what pushes the inclination towards zero with Campbell's approach when $a_{1} \sin i$ gets small is also responsible for pushing the Thiele-Innes $K_{1}$ towards a large value.

\subsection{The law of the semi-major axis}

Since we cannot rely on the inclination, we now turn to the semi-major axis. It is indeed their values together with a small spectroscopic $a_{1} \sin i$ which pushed the inclinations towards small values in the Campbell approach.

Han et al. (2001) selected the large secondary masses using the ratio $\frac{a_{\mathrm{a}}}{\sigma_{\mathrm{a}}}$ so the question is whether large values of this ratio are expected or not. We proceed by simulations since one cannot easily recover the true $a_{1}$ distribution either by assuming a Rayleigh-Rice law (Halbwachs et al. 2000b) or with an approximation formula (Han et al. 2001). The Rayleigh-Rice law was shown by simulations to be globally compatible with the observed $a_{1}$ on a sample of stars, but what we need here should be done on a star by star basis.

For each star, we assume the extreme case that all true $a_{1}$ are minimum, corresponding to $i=90^{\circ}$, the other orbital and astrometric parameters being those obtained in the solution with the real data. We then draw at random Gaussian Hipparcos abscissae around their expected value and a Campbell solution is computed. Using 1000 such simulations we obtain the empirical probability for 


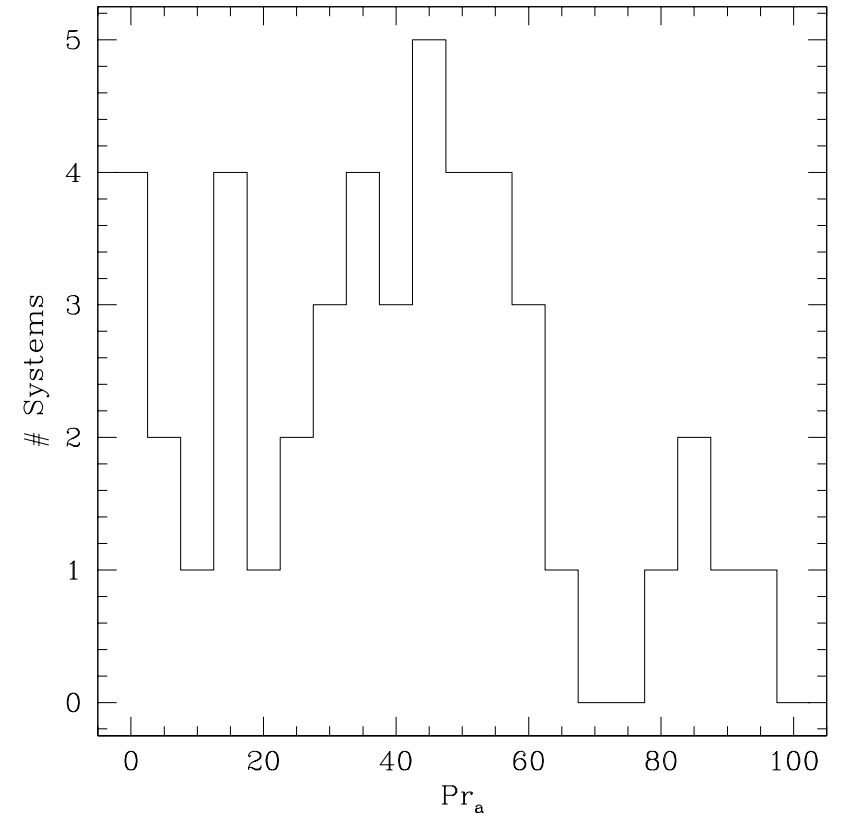

Fig. 4. Probability that simulated $\frac{a_{\mathrm{a}}}{\sigma_{\mathrm{a}}}\left(\right.$ assuming $i=90^{\circ}$ ) are larger than the observed one for the 46 -sample.

each star that the $\frac{a_{\mathrm{a}}}{\sigma_{\mathrm{a}}}$ ratio is larger than the observed one (column labelled $\operatorname{Pr}_{\mathrm{a}}$ in Table 1).

As can be seen in Fig. 4, this probability is not uniform (significance level 3\%) which is not surprising since the simulations assumed no outlier, no other unmodelled astrometric perturbation and $i=90^{\circ}$. The main point however is that there is no indication of a significant fraction of the real sample with a large $\frac{a_{\mathrm{a}}}{\sigma_{\mathrm{a}}}$ ratio, which would correspond to small $\operatorname{Pr}_{\mathrm{a}}$ in the simulations. There are six orbits with a probability smaller than $5 \%$, but this is not completely unexpected since this has a $3 \%$ probability to occur in a 46-sample. Among these stars, the result of $\rho \mathrm{CrB}$ and HD 195019 are however less likely $($ prob $=0.01 \%)$ to occur simultaneously. This could indicate a significant orbit or other astrometric perturbations for these stars.

This exercise shows, in some sense, that a whole sample made of edge-on orbits would be more probable than the large fraction of face-on orbits which are obtained by the direct Campbell approach. Edge-on orbits for all stars are of course unrealistic, and this shows merely that there is no orbital information in the Hipparcos data for the sample as a whole.

\section{Screening the astrometric solutions}

At this point, it is clear that there is no indication in the planetary sample as a whole of a significant fraction of low inclinations/large secondary mass. Due to the increasing number of planetary candidates, it is however expected on a statistical basis that a bunch of them may have a mass much larger than the minimum mass.

Since there is evidence that a direct use of the orbital parameters (without taking their statistical distribution into account) may lead to incorrect conclusions, we need a set of tests which can tell us whether the Campbell results are correct or not. Since by construction the Campbell analysis is consistent with the spectroscopic orbit, this is where the Thiele-Innes approach reveals useful.

The tests described below intend to show whether an orbital model improves the fit of the Hipparcos data or not, whether the Thiele-Innes elements are consistent with Campbell's ones or not and, finally, whether the astrometric and spectroscopic orbits agree with each other or not. This is an essential point: before combining the spectroscopic and astrometric data, as done in the Campbell approach, one has to be sure that both solutions are compatible. If all these tests give a positive result, then the orbital elements obtained with the Campbell approach may be trusted.

In the other cases, one may either conclude that the orbital elements were obtained by chance only, or that there is a perturbation in the astrometric solution due e.g. to another companion. The presence of other companions around several stars hosting planetary candidates can be detected from a radial velocity residual trend (Fischer et al. 2001) and some of these companions are being discovered by adaptive optics: HD 114762, $\tau$ Boo (LLoyd et al. 2000) and Gl 86 (Els et al. 2001). Arenou $\&$ Palasi (2000) combined the spectroscopic data with the Hipparcos and Tycho2 data (Høg et al. 2000) for the planetary candidates and the use of long term proper motion gave a hint of possible other companions (period larger than several decades).

The spectroscopic orbital elements are generally precise. So, from now on, we treat them as constants. As we have seen, the statistical distributions of the semi-major axis and of the inclination are not trivial, so our tests must be based on better known parameters. This is the case for the $A, B, F, G$ Thiele-Innes parameters: obtained by a linear least-squares solution, they may be considered Gaussian around 0, as has been confirmed by direct simulations. Concerning real Hipparcos data, it has been shown that the errors on the astrometric parameters could be considered Gaussian (Arenou et al. 1995). So the normality hypothesis for the Thiele-Innes elements is not just a convenient hypothesis but is plainly justified. Of course, a departure from normality may be due to outliers. Apart from possible instrumental errors, however, outliers may be normal observations when a good orbital model is used in place of the standard "single star" model adopted for the major part of the Hipparcos stars.

\subsection{Need for an orbital motion}

As pointed out by Pourbaix (2001), none of the Campbelllike orbital solutions but four does improve the fit of the Hipparcos intermediate astrometric data (IAD). Could it be different with a Thiele-Innes solution? Do the two 
Table 1. List of systems (Pourbaix 2001) and the statistical tests. $\operatorname{Pr}_{\mathrm{a}}=$ Probability to get a $a_{\mathrm{a}} / \sigma_{\mathrm{a}}$ larger than the observed value assuming $i=90^{\circ} ; \operatorname{Pr}_{1}=\operatorname{Pr}\left(\hat{F}_{\mathrm{C}}>F(2, N-7)\right.$ ) (Pourbaix 2001); $\operatorname{Pr}_{2}=\operatorname{Pr}(\hat{F}>F(4, N-9)) ; \operatorname{Pr}_{3}=\operatorname{Pr}\left(\chi_{4 \mathrm{~S}}^{2}>\chi^{2}(4)\right)$; $\operatorname{Pr}_{4}=\operatorname{Pr}\left(\chi_{4 \mathrm{C}}^{2}>\chi^{2}(4)\right)$; The Campbell approach may be accepted when the conditions $\operatorname{Pr}_{1}<5 \%, \operatorname{Pr}_{2}<5 \%, \operatorname{Pr}_{3}<5 \%$, $|D|<\approx 2, \operatorname{Pr}_{4}>5 \%, \operatorname{Pr}_{5}>5 \%$ are fulfilled.

\begin{tabular}{|c|c|c|c|c|c|c|c|c|c|}
\hline HIP & $\mathrm{HD} /$ name & Ref & $\begin{array}{l}\mathrm{Pr}_{\mathrm{a}} \\
(\%)\end{array}$ & $\begin{array}{l}\mathrm{Pr}_{1} \\
(\%)\end{array}$ & $\begin{array}{l}\mathrm{Pr}_{2} \\
(\%)\end{array}$ & $\begin{array}{l}\mathrm{Pr}_{3} \\
(\%)\end{array}$ & $D$ & $\begin{array}{l}\mathrm{Pr}_{4} \\
(\%)\end{array}$ & $\begin{array}{l}\operatorname{Pr}_{5} \\
(\%)\end{array}$ \\
\hline 1292 & GJ 3021 & Naef et al. (2000) & 67 & 66 & 89 & 89 & 0.24 & 99 & 87 \\
\hline 5054 & 6434 & Queloz et al. (2000a) & 14 & 51 & 12 & 33 & 0.77 & 44 & 15 \\
\hline $7513 \mathrm{~b}$ & $v$ And $\mathrm{b}$ & Butler et al. (1999) & 55 & 62 & 52 & 50 & -0.38 & 66 & 30 \\
\hline $7513 c$ & $v$ And $\mathrm{c}$ & Butler et al. (1999) & 16 & 23 & 14 & 14 & 0.93 & 41 & 14 \\
\hline $7513 d$ & $v$ And $\mathrm{d}$ & Butler et al. (1999) & 37 & 20 & 25 & 25 & -0.25 & 71 & 34 \\
\hline 8159 & 10697 & Vogt et al. (2000) & 2 & 6 & 19 & 5 & 0.53 & 89 & 61 \\
\hline 9683 & 12661 & Fischer et al. (2001) & 79 & 36 & 65 & 51 & 0.24 & 96 & 73 \\
\hline 10138 & Gl 86 & Queloz et al. (2000b) & 39 & 75 & 64 & 64 & 0.44 & 72 & 36 \\
\hline 12048 & 16141 & Marcy et al. (2000) & 50 & 46 & 59 & 59 & 0.59 & 86 & 52 \\
\hline 12653 & HR 810 & Kürster et al. (2000) & 28 & 20 & 28 & 23 & 0.34 & 95 & 73 \\
\hline 14954 & 19994 & Queloz et al. (2000a) & 42 & 40 & 60 & 61 & 0.21 & 92 & 63 \\
\hline 16537 & $\epsilon$ Eri & Hatzes et al. (2000) & 36 & 44 & 71 & 59 & -0.24 & 95 & 71 \\
\hline 26381 & 37124 & Vogt et al. (2000) & 86 & 55 & 58 & 23 & 0.03 & 41 & 13 \\
\hline 27253 & 38529 & Fischer et al. (2001) & 3 & 8 & 8 & 1 & -2.07 & 48 & 26 \\
\hline 31246 & 46375 & Marcy et al. (2000) & 46 & 46 & 93 & 0 & 0.38 & 0 & 0 \\
\hline 33719 & 52265 & Butler et al. (2000) & 54 & 59 & 77 & 74 & 0.11 & 93 & 66 \\
\hline 43587 & $55 \mathrm{Cnc}$ & Butler et al. (1997) & 30 & 30 & 13 & 12 & -0.65 & 32 & 9 \\
\hline 47007 & 82943 & Naef et al. (2001) & 25 & 61 & 68 & 61 & 0.04 & 81 & 46 \\
\hline $47202 b$ & $83443 b$ & Mayor et al. (2000) & 5 & 13 & 15 & 9 & -0.07 & 52 & 20 \\
\hline $47202 \mathrm{c}$ & $83443 \mathrm{c}$ & Mayor et al. (2000) & 2 & 3 & 8 & 4 & -1.04 & 77 & 40 \\
\hline 50786 & 89744 & Korzennik et al. (2000) & 86 & 35 & 62 & 42 & 0.60 & 93 & 69 \\
\hline 52409 & 92788 & Queloz et al. (2000a) & 26 & 22 & 31 & 27 & -0.17 & 74 & 38 \\
\hline 53721 & $47 \mathrm{UMa}$ & Butler \& Marcy (1996) & 97 & 86 & 98 & 96 & -0.12 & 99 & 90 \\
\hline 64426 & 114762 & Marcy et al. (1999) & 56 & 41 & 52 & 57 & 0.21 & 86 & 52 \\
\hline 65721 & 70 Vir & Marcy \& Butler (1996) & 38 & 42 & 69 & 59 & -0.58 & 95 & 71 \\
\hline 67275 & $\tau$ Boo & Butler et al. (1997) & 60 & 55 & 74 & 34 & -0.72 & 25 & 14 \\
\hline 68162 & 121504 & Queloz et al. (2000a) & 21 & 27 & 47 & 35 & -0.51 & 87 & 54 \\
\hline 72339 & 130322 & Udry et al. (2000a) & 46 & 61 & 40 & 29 & 0.44 & 44 & 15 \\
\hline 74500 & 134987 & Vogt et al. (2000) & 59 & 55 & 84 & 90 & 0.26 & 99 & 90 \\
\hline 78459 & $\rho \mathrm{CrB}$ & Noyes et al. (1999) & .2 & .2 & 1 & .1 & -0.34 & 99 & 87 \\
\hline 79248 & 14 Her & Udry et al. (2000b) & 36 & 25 & 37 & 44 & 0.19 & 86 & 45 \\
\hline $89844 b$ & $168443 \mathrm{~b}$ & Udry et al. (2000b) & 44 & 50 & 96 & 77 & 0.22 & 95 & 75 \\
\hline $89844 \mathrm{c}$ & $168443 \mathrm{c}$ & Udry et al. (2000b) & 88 & 60 & 92 & 63 & -0.17 & 98 & 91 \\
\hline 90485 & 169830 & Naef et al. (2001) & 13 & 10 & 30 & 37 & -0.02 & 97 & 79 \\
\hline 93746 & 177830 & Vogt et al. (2000) & 15 & 19 & 28 & 33 & 0.69 & 81 & 46 \\
\hline 96901 & 16 Cyg B & Cochran et al. (1997) & 44 & 41 & 65 & 67 & -0.52 & 95 & 70 \\
\hline 97336 & 187123 & Vogt et al. (2000) & 34 & 17 & 46 & 62 & -0.22 & 99 & 95 \\
\hline 98714 & 190228 & Sivan et al. (2000) & 12 & 9 & 24 & 22 & -0.27 & 94 & 67 \\
\hline 99711 & 192263 & Vogt et al. (2000) & 30 & 73 & 48 & 20 & -2.48 & 30 & 8 \\
\hline 100970 & 195019 & Vogt et al. (2000) & .5 & 0 & 0 & 0 & -4.68 & 6 & 1 \\
\hline 108859 & 209458 & Mazeh et al. (2000) & 48 & 19 & 15 & 8 & -2.08 & 40 & 13 \\
\hline 109378 & 210277 & Vogt et al. (2000) & 53 & 41 & 72 & 66 & 0.00 & 99 & 97 \\
\hline 113020 & Gl 876 & Delfosse et al. (1998) & 49 & 49 & 43 & 53 & 0.32 & 74 & 37 \\
\hline 113357 & $51 \mathrm{Peg}$ & Mayor \& Queloz (1995) & 46 & 65 & 86 & 79 & -0.13 & 99 & 95 \\
\hline 113421 & 217107 & Vogt et al. (2000) & 61 & 54 & 60 & 68 & 0.62 & 86 & 53 \\
\hline 116906 & 222582 & Vogt et al. (2000) & 52 & 10 & 17 & 28 & 0.32 & 82 & 47 \\
\hline
\end{tabular}

additional degrees of freedom significantly reduce the $\chi^{2}$ ? The quantity

$\hat{F}=\frac{N-9}{4} \frac{\chi_{\mathrm{S}}^{2}-\chi_{\mathrm{T}}^{2}}{\chi_{\mathrm{T}}^{2}}$ follows a $F$-distribution with $(4, N-9)$ degrees of freedom (Bevington \& Robinson 1992). $N$ denotes the number of data points and $\chi_{\mathrm{S}}^{2}$ and $\chi_{\mathrm{T}}^{2}$ are the value of the $\chi^{2}$ with the 5-parameter (single star) model and Thiele-Innes' orbital model respectively. We reject the null hypothesis "no orbital wobble present in the IAD" if the probability of 
getting $\hat{F}$ larger than $F(4, N-9)$ is lower than $5 \%$. This $P$-value is given as $\operatorname{Pr}_{2}$ in Table 1 where we also recall as $\operatorname{Pr}_{1}$ the analogous probability obtained by Pourbaix (2001) for the Campbell approach.

\subsection{Significance of $A, B, F$, and $G$}

Getting a substantial reduction of the $\chi^{2}$ with the ThieleInnes model does not necessary mean the four constants are significantly different from 0 . In order to assess that, we compute

$\chi_{4 \mathrm{~S}}^{2}=\boldsymbol{X}^{\mathrm{t}} \boldsymbol{V}^{-1} \boldsymbol{X}$

where $\boldsymbol{X}=(A, B, F, G)$ and $\boldsymbol{V}$ is the covariance matrix of $A, B, F$, and $G$. "t" denotes the transposition. $\chi_{4 \mathrm{~S}}^{2}$ follows a $\chi^{2}$-distribution with 4 degrees of freedom when there is no orbital information. When all formal errors on ThieleInnes parameters are identical, this $\chi_{4 \mathrm{~S}}^{2}$ is proportional to $a_{\mathrm{a}}$. We then reject the null hypothesis " $a_{\mathrm{a}}$ (mas) is significantly different from 0 " if the probability that $\chi_{4 S}^{2}$ exceeds $\chi^{2}(4)$ is less than $5 \%$. This $P$-value $\operatorname{Pr}_{3}$ is given as in Col. 7 of Table 1.

\subsection{Consistency between spectroscopy and astrometry}

Testing such a consistency is a bit tricky. Indeed, the nature of the information supplied with by both sides is essentially different. The astrometry should however be able to recover $a \sin i$ and $\omega_{1}$, both given by spectroscopy. There are a few useful relations based on the Thiele-Innes elements such as

$$
\begin{aligned}
L & =A^{2}+B^{2}-\left(F^{2}+G^{2}\right)=\left(a_{\mathrm{a}} \sin i\right)^{2} \cos \left(2 \omega_{1}\right) \\
\text { and } M & =A^{2}+B^{2}+F^{2}+G^{2}=2 a_{\mathrm{a}}^{2}-\left(a_{\mathrm{a}} \sin i\right)^{2}
\end{aligned}
$$

that can be used to build up a test. For instance,

$$
D=\frac{L_{\mathrm{l}}-L_{\mathrm{r}}}{2 \sigma \sqrt{M_{\mathrm{r}}+2 \sigma^{2}}}
$$

follows a distribution of null expectation and unit variance. $L_{1}$ denotes the left-hand side of the definition of $L$, i.e. based on the Thiele-Innes constants only while $L_{\mathrm{r}}$ and $M_{\mathrm{r}}$ are computed from the spectroscopic elements and the parallax. In the latter, the true $a_{\mathrm{a}}$ is unfortunately unknown, so it is derived from the Campbell-like solution. $\sigma$ stands for the mean standard errors of $A, B, F$, and $G$. Because we neglect the correlation between the Thiele-Innes constants, and with the $a_{\mathrm{a}}$ deduced from the Campbell analysis, $D$ is not Gaussian, and $|D|<2$ does not strictly correspond to a $95 \%$ confidence level. Indeed, as can be seen in Table 1 , the distribution of $D$ is leptokurtic: $a_{\mathrm{a}}$ being overestimated, $|D|$ is generally underestimated. A large value of $|D|$ is then a good indication that the astrometric solution is not consistent with the spectroscopic orbit.

\subsection{Consistency between Thiele-Innes' and Campbell's solutions}

Since both methods are applied to the same data set, they should also give similar results. We have already shown that edge-on orbits are consistent with pure noise signal when fitted with the Thiele-Innes parameters while Pourbaix (2001) got similar results for face-on orbits with Campbell's elements.

Here also, designing a test may be difficult, especially because the distribution of $\Omega$ is anything but symmetrical. So, instead of comparing the two sets of Campbell elements, we compute their Thiele-Innes expressions. We then build

$\chi_{4 \mathrm{C}}^{2}=\boldsymbol{Y}^{\mathrm{t}} \boldsymbol{V}^{-1} \boldsymbol{Y}$

where $\boldsymbol{Y}=\left(A-A_{\mathrm{C}}, B-B_{\mathrm{C}}, F-F_{\mathrm{C}}, G-G_{\mathrm{C}}\right), \boldsymbol{V}$ has the same meaning as in $\chi_{4 \mathrm{~S}}^{2}$ (Eq. (4)) and $\left(A_{\mathrm{C}}, B_{\mathrm{C}}, F_{\mathrm{C}}, G_{\mathrm{C}}\right)$ are computed from the Campbell elements. If we neglect the correlations between Campbell and Thiele-Innes, the quantity $\chi_{4 \mathrm{C}}^{2}$ follows a $\chi^{2}$-distribution with 4 degrees of freedom. We reject the null hypothesis "Thiele-Innes" and Campbell's approaches yield consistent solutions' if the probability that $\chi_{4 \mathrm{C}}^{2}$ exceeds $\chi^{2}(4)$ is less than $5 \%$. That probability $\mathrm{Pr}_{4}$ is given in Table 1 . Because of the correlations between the $A, B, F, G$ obtained by both methods, a rejection at a $5 \%$ level will occur in much less than $5 \%$ of the cases. In fact, $\chi_{4 \mathrm{C}}^{2}$ is probably closer to a $\chi^{2}$ distribution with 2 degrees of freedom, so this test is less discriminant than the next one.

\subsection{A small inclination}

Finally, one may answer both whether a small inclination is present or whether the astrometric solution is compatible with the spectroscopic one, still using the Thiele-Innes elements as an external control. Specifically,

$$
\begin{aligned}
& A-G=\varpi a_{1} \sin i \cos \left(\Omega-\omega_{1}\right) \tan \left(\frac{i}{2}\right) \\
& B+F=\varpi a_{1} \sin i \sin \left(\Omega-\omega_{1}\right) \tan \left(\frac{i}{2}\right)
\end{aligned}
$$

and

$A+G=\varpi a_{1} \sin i \cos \left(\Omega+\omega_{1}\right) \cot \left(\frac{i}{2}\right)$

$B-F=\varpi a_{1} \sin i \sin \left(\Omega+\omega_{1}\right) \cot \left(\frac{i}{2}\right)$.

In the case of the planetary candidates, $a_{\mathrm{a}} \sin i$ is small, and, if the inclination is really small, then the right-hand sides of Eq. (9) are null; when the inclination is found to be near $180^{\circ}$, the Eq. (10) should be $\approx 0$. We test whether Eq. (9) (if $\cos i_{\mathrm{C}}>0$ ) or Eq. (10) (if $\cos i_{\mathrm{C}}<0$ ) are verified. If $\boldsymbol{Z}$ denotes this two-dimensional vector, and $\boldsymbol{W}$ its covariance matrix computed using the covariance matrix of $(A, B, F, G)$, then

$\chi_{2}^{2}=\boldsymbol{Z}^{\mathrm{t}} \boldsymbol{W}^{-1} \boldsymbol{Z}$ 
should follow a $\chi^{2}$ with two degrees of freedom under the null hypothesis of a Thiele-Innes solution compatible with the Campbell/spectroscopic solution. The $P$-value $\operatorname{Pr}_{5}$ is given in Table 1 . We will reject the compatibility between Thiele-Innes and Campbell if $\operatorname{Pr}_{5}$ is smaller than 5\%.

\subsection{Discussion}

Since the proposed tests are not independent, we cannot compute an overall probability. While the first three tests check whether the astrometric solution is firmly established, the last three ones (useless in the case of a "noise only" orbit) assess the agreement between the astrometric and spectroscopic orbits. In fact $\operatorname{Pr}_{1}$ and $\operatorname{Pr}_{5}$ would probably be enough for investigations of future planetary candidates, provided that a signal is indeed present at the spectroscopic period. It appears that, with the exception of $\rho \mathrm{CrB}$, almost all stars in the present sample fail to at least two of the proposed tests.

Under the assumption of a "noise only" orbit, one expects that the computed probabilities are uniform on this sample. Once again, a departure of the Hipparcos residuals from strict normality (e.g. due to outliers or long period companions) could produce spurious solutions which in turn would contaminate the probabilities. Nevertheless, using a Kolmogorov test, the uniform null hypothesis is accepted for $\operatorname{Pr}_{2}(P$-value $=88 \%), \operatorname{Pr}_{3}(7 \%), \operatorname{Pr}_{5}(98 \%)$.

If this was necessary, this terminates the demonstration that the Hipparcos data mainly contains noise. This does not mean that there is no brown or red stars unfortunately interpreted as planet (apart $\rho \mathrm{CrB}$ ), but rather that Hipparcos is of little help to demonstrate it, to the contrary of the Han et al. (2001) analysis.

\section{Individual stars}

\subsection{Validity of $i_{C}$ with $H D 209458$}

HD 209458 (HIP 108859) is, so far, the only case where the inclination is accurately known. Indeed, transits of the planet have been monitored since 1999 (Charbonneau et al. 2000), thus setting a strong constraint on the inclination: $i=86.1 \pm 1.6^{\circ}$ (Mazeh et al. 2000).

Hipparcos observed that system and it has been noticed later on that it did detect the planet... thanks to its photometric signature (Söderhjelm 1999; Robichon \& Arenou 2000; Castellano et al. 2000). However, that result does not mean the astrometric signature is also in the IAD. Thus, when $i_{\mathrm{C}}$ and $\Omega$ are fitted together with the five astrometric parameters, the inclination is $i_{\mathrm{C}}=0.019 \pm 0.0097^{\circ}$. Fitting the Thiele-Innes elements yields an inclination $i_{\mathrm{T}}=76 \pm 18^{\circ}$.

It appears that the 'noise only' null hypothesis cannot be rejected as indicated by three tests $\left(\mathrm{Pr}_{1}, \mathrm{Pr}_{2}\right.$ and $\left.\mathrm{Pr}_{3}\right)$. Moreover the hypothesis of a small inclination has a small $P$-value $\left(\operatorname{Pr}_{5}=13 \%\right)$. On this real case where the true orbit is known, the proposed tests thus seems discriminating enough to detect wrong Campbell solutions.
Table 2. Campbell analysis with different trial periods on HD 10697.

\begin{tabular}{r|r|c}
\hline $\begin{array}{r}\text { Period } \\
\text { (days) }\end{array}$ & $\begin{array}{r}a_{\mathrm{a}} \\
(\mathrm{mas})\end{array}$ & GOF \\
\hline 700 & $1.9 \pm 0.7$ & 1.31 \\
800 & $1.9 \pm 0.7$ & 1.28 \\
900 & $1.9 \pm 0.7$ & 1.27 \\
1000 & $2 . \pm 0.7$ & 1.32 \\
1072 & $2.1 \pm 0.7$ & 1.28 \\
1100 & $2.2 \pm 0.8$ & 1.25 \\
1200 & $2.7 \pm 0.9$ & 1.24 \\
1300 & $3 \pm 1$ & 1.29 \\
\hline
\end{tabular}

\section{2. $\rho \operatorname{Cr} B$}

The suggestion that the secondary could in fact be an M dwarf was made by Gatewood et al. (2001) using both Hipparcos and MAP (Gatewood 1987) data, and the solution in the Hipparcos Catalogue was already detected as orbital with a period twice the spectroscopic period. As shown by Gatewood et al. (2001) a periodogram exhibits a strong signal at this period.

This is the only star in the "planetary" sample which succeeds to all tests: while the hypothesis of a "noise only" orbit is significantly rejected by the three first tests, there is no indication that the spectroscopic, Campbell and Thiele-Innes orbits could be inconsistent, using the other tests. Consequently, the main result of Gatewood et al. (2001) may not be rejected.

\section{3. $H D 10697$}

Since the minimum reflex semi-major axis is about 0.4 mas, and thus suggests that Hipparcos could give a hint of the true semi-major axis, Zucker \& Mazeh (2000) combined the astrometric and spectroscopic data and obtained $a_{\mathrm{a}}=2.1 \pm 0.7$ mas, corresponding to a $38 \pm 13 M_{\mathrm{J}}$ mass for the secondary. This result would indicate that the secondary is in the brown dwarf domain.

Although the signal should be present to some extent in the Hipparcos data, a periodogram based on the unitweight error of the Thiele-Innes solution shows no signal at the 1072 days spectroscopic period (Fig. 5). Instead a 345 days period would be more likely, but would have been seen in the spectroscopic data.

Repeating this periodogram with the Campbell analysis is shown in Table 2. While the obtained semi-major axis increases with trial period, the inclination decreases accordingly, the same large signal to noise $\approx 3$ is obtained, and the goodness of fit (GOF) is not improved at the spectroscopic period. The Campbell analysis simply adjusts itself to the constrained spectroscopic elements, without showing any sensible information in the astrometric data.

This explains the negative results of our tests in Table 1. Due to the size of the astrometric residuals (partly independent from the orbital perturbation), the obtained semi-major axis is probably overestimated, but it is 


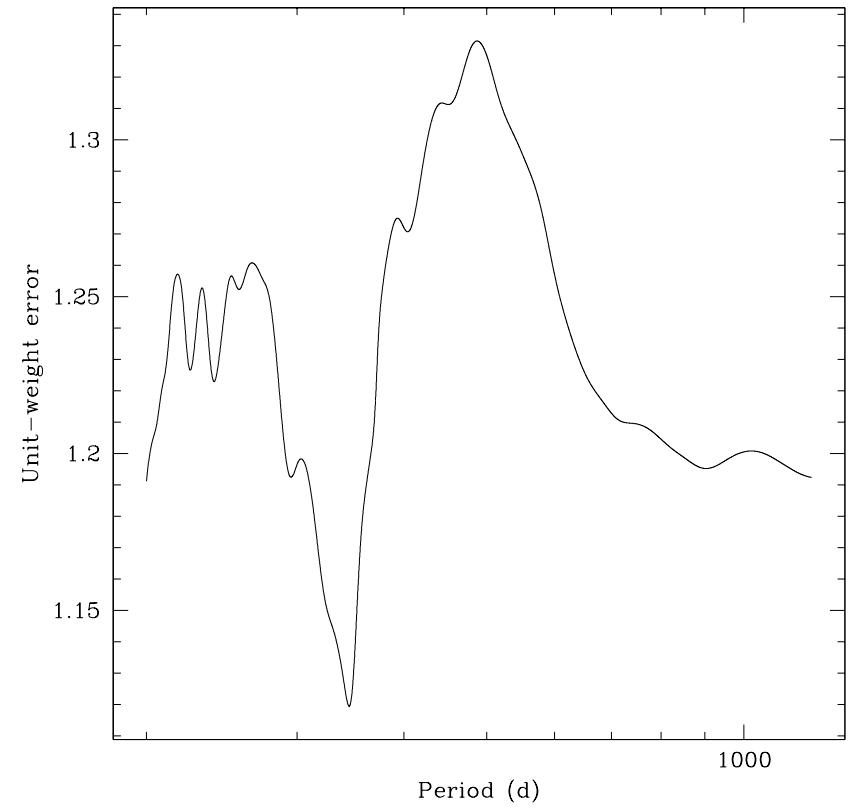

Fig. 5. Periodogram of HD 10697 assuming $e$ and $T$ from the known companion.

difficult to know to which extent. Consequently the exact mass of the secondary cannot be obtained at face value using a combination of the Hipparcos data with the spectroscopic orbital elements.

\section{4. $H D 195019$}

This is probably a typical case of how one can be mislead by a direct Campbell analysis: the fit of the IAD is significantly improved by the orbital model but fails the last two tests, i.e. the astrometric orbit is clearly not consistent with the spectroscopic one. The periodogram based on the orbital parameters of the known companion (Fig. 6) reveals a peak at 68 days and nothing around 18 days (the orbital period of the planetary companion).

We cannot find any satisfactory solution by fitting the 12 parameters with a global optimisation technique. We end up with a minimum $\chi^{2}$ which corresponds to $e \approx 1$ and a total correlation between $F, G$, and $e$. Even if that solution does minimise $\chi^{2}(12)$, it is physically very unlikely.

Instead of fitting the 12 parameters in once, one can use the periodogram and build up the solution by adding the parameters one by one. Thus, by first fixing $P$ to 67.935 d, one derives $T_{0}=$ JD $2,448,296.8$ which yields the lowest value of the unit-weight error. The resulting orbit would have an inclination of $65 \pm 18^{\circ}$ and $K_{1}=$ $24 \pm 8 \mathrm{~km} \mathrm{~s}^{-1}$. Unlike the orbit of the known companion, this solution successfully passes the last two tests. However such a solution would have been present in the radial velocity residuals.

Another explanation could originate in the fact that HD 195019 is known since 1881 as a binary star, with a B component $4.5^{\prime \prime}$ apart $\left(3.5^{\prime \prime}\right.$ in 1988$)$ with a position angle

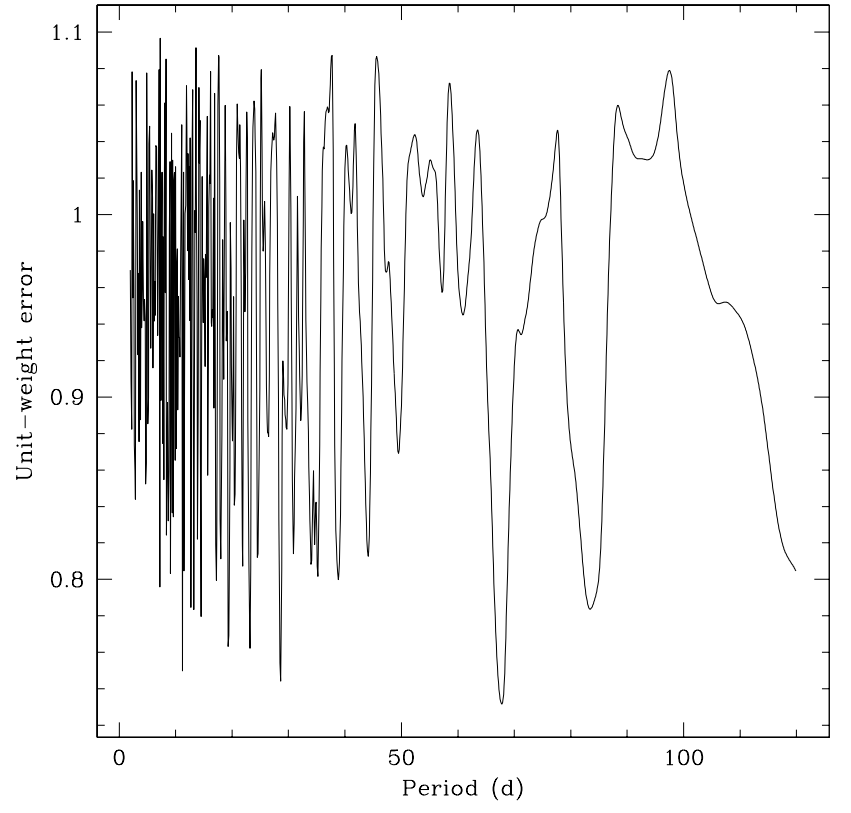

Fig. 6. Periodogram of HD 195019 assuming $e$ and $T$ from the known companion.

of $323^{\circ}$ (resp. $330^{\circ}$ ), from the WDS Catalog. Despite this fact, it has been dealt as a single star in the Hipparcos Catalogue (although a "suspected non-single star" flag is set), because no convincing double star solution could be used. Although $\approx 3$ mag fainter, the influence of the secondary may have produced astrometric residuals (the projected separation varying with the satellite scan orientation) which could be wrongly attributed to the reflex motion due to the low-mass companion, especially if the companion had a significant motion during the Hipparcos observation period.

\section{5. $H D 38529$}

A periodogram reveals nothing special at the 14-day spectroscopic period. However, this is another star for which Han et al. (2001) suggest that the secondary could be an M dwarf. They also quote that this star received an acceleration solution in the Hipparcos Catalogue.

Before looking to the small signal due to the spectroscopic companion, one has to remove the effect due to the long period companion. When the acceleration terms in time are thus subtracted, the semi-major axis of the Campbell solution becomes $1.3 \pm .7$ mas, i.e. not significant, whereas it was $2.1 \pm .6$ mas before; when a cubic instead of quadratic term in time is accounted for, $a_{\mathrm{a}}=.9 \pm .7$ mas.

The influence of a long-period companion, also detected in the spectroscopic data (Fischer et al. 2001), is thus a much more natural explanation to the size of the Hipparcos residuals than a tiny inclination for the short period companion. 


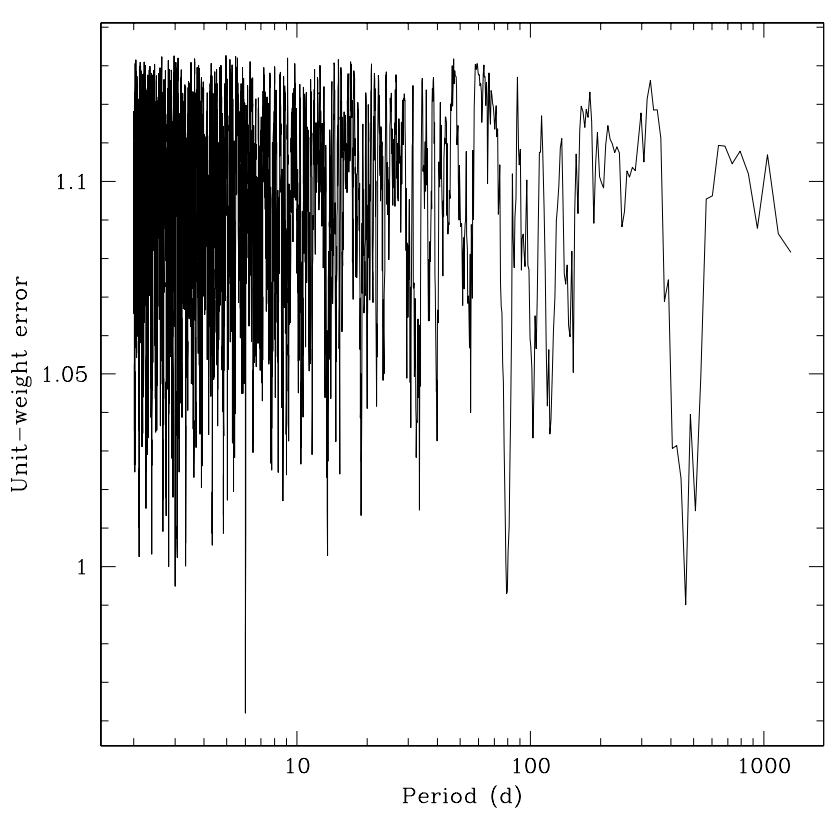

Fig. 7. Periodogram of HD 83443 assuming $e$ and $T$ from the second companion.

\section{6. $H D 83443$}

There is also no signal around the periods of the two planetary candidates $(\approx 3$ and 30 days) in the Hipparcos data. Instead a period of 6,79 or 462 days (Fig. 7) would be much more likely, but it would clearly have been seen in the spectroscopic data. An unmodelled astrometric perturbation due to a longer period companion would provide a more logical explanation, since there is a possible trend in the radial velocity residuals (S. Udry, private communication).

Still, based on the tests we presented, the astrometric solution for HD 83443c was about to be accepted. The reason for this is the large $a_{\mathrm{a}}=2 \pm 0.7$ mas.

One may ask whether any other period could have given the same signal as the one obtained with a 30 days period. All above mentioned tests were based on normality assumptions about the astrometric residuals but another, non-parametrical test may also be used. A periodogram, based on the amplitude of the signal (root-sumsquare of the 4 Thiele-Innes parameters) has been done: using 5000 trial periods between 2 and 1500 days, equally spaced in frequency, a Thiele-Innes solution is performed. It happens that $30 \%$ of the trial periods give an amplitude larger than the one obtained at the period of the spectroscopic companion. In other words, the astrometric result has no special meaning.

\section{Conclusion}

In summary, the available Hipparcos data is not precise enough to show that a significant part of the planetary candidates could be of stellar nature.

Although we expect a very small number of small inclinations in the putative extrasolar planets sample, the inclinations derived using the Campbell elements together with the Hipparcos data are pushed towards unreasonably small values. As we have shown, this is not due to an observational selection but rather to the employed reduction method and probably also due to the presence of longer period, unresolved companions.

Though the Thiele-Innes alternative method gives inclinations more in line with what is generally expected from the spectroscopic detection, the estimated astrometric semi-major axis still does not provide a statistically unbiased estimate of the true reflex motion. Indeed, in the case of pure noise in the astrometric data, the Campbell approach would give inclinations biased towards $0^{\circ}$ while Thiele-Innes recovers inclinations biased towards $90^{\circ}$. So, none of the two methods can be trusted, except when it can be shown that they agree together, which is the basis of one of the tests we propose. The other tests determine whether the astrometric orbit is significant or might result from noise only.

It must be pointed out that the bias on the semi-major axis and inclination only occurs for negligible orbital wobbles. When a true orbital information is present in the Hipparcos astrometric data, then the orbital parameters are much better behaved. For instance, we may question whether the rejection of putative brown dwarfs into the stellar domain by the Halbwachs et al. (2000b) study was justified. The stars HIP 13769, 19832, 62145, 63366 and 113718 which were found with a mass above the H-burning limit with a $2 \sigma$ significance and HIP 77152, one $\sigma$ above, have been tested using the tests described above. All these stars succeed to all tests, apart from HIP 19832 which fails to the last test (significance $=3 \%$, this may be by chance only since the reality of the orbit is clear, as seen e.g. by periodogram). The main conclusion of Halbwachs et al. (2000b), the deficit of short period brown dwarf secondaries, is thus confirmed by this analysis.

In contrast, in the sample of putative planets, there is no indication that a significant fraction of secondaries could be brown or red dwarfs, with the possible exception of $\rho \mathrm{CrB}$. Is that to say that the Hipparcos astrometry is useless for the extrasolar planet study? Not completely, since at least an upper limit on the secondary mass may be obtained, and Hipparcos may also give a hint of other, longer period companions. For a satellite which was originally planned to get positions, proper motions and parallaxes, this is not negligible.

Acknowledgements. We thank J.-L. Halbwachs, M. Mayor and S. Udry for their useful comments. DP thanks the National Aeronautics and Space Administration which partially supported this work via grant NAG5-6734.

\section{References}

Arenou, F., Lindegren, L., Froeschle, M., et al. 1995, A\&A, 304,52

Arenou, F., \& Palasi, J. 2000, in Planetary Systems in the Universe: Observation, Formation and Evolution, IAU 
Symposium 202, ASP Conference Series \#, ed. A. J. Penny, P. Artymowicz, A. M. Lagrange, \& S. S. Russell

Batten, A. H., Fletcher, J. M., \& McCarthy, D. G. 1989, Publ. Dom. Ap. Obs., 17, 1

Bevington, P. R., \& Robinson, D. K. 1992, Data reduction and error analysis for the physical sciences, 2nd ed. (McGrawHill)

Butler, R. P., \& Marcy, G. W. 1996, ApJ, 464, L153

Butler, R. P., Marcy, G. W., Fischer, D. A., et al. 1999, ApJ, 526,916

Butler, R. P., Marcy, G. W., Williams, E., Hauser, H., \& Shirts, P. 1997, ApJ, 474, L115

Butler, R. P., Vogt, S. S., Marcy, G. W., et al. 2000, ApJ, 545, 504

Castellano, T., Jenkins, J., Trilling, D. E., Doyle, L., \& Koch, D. 2000, ApJ, 532, L50

Charbonneau, D., Brown, T. M., Latham, D. W., \& Mayor, M. 2000, ApJ, 529, L45

Cochran, W. D., Hatzes, A. P., Butler, R. P., \& Marcy, G. W. 1997, ApJ, 483, 457

Delfosse, X., Forveille, T., Mayor, M., et al. 1998, A\&A, 338, L67

Duquennoy, A., \& Mayor, M. 1991, A\&A, 248, 485

Els, S. G., Sterzik, M. F., Marchis, F., et al. 2001, A\&A, 370, L1

ESA. 1997, The Hipparcos and Tycho Catalogues (ESA SP-1200)

Fischer, D. A., Marcy, G. W., Butler, R. P., et al. 2001, ApJ, accepted

Gatewood, G., Han, I., \& Black, D. 2001, ApJ, 548, L61

Gatewood, G. D. 1987, AJ, 94, 213

Halbwachs, J. L., Arenou, F., Mayor, M., \& Udry, S. 2000a, in The Formation of Binary Stars, Poster Proceedings of IAU Symposium No. 200, ed. B. Reipurth, \& H. Zinnecker, 135

Halbwachs, J. L., Arenou, F., Mayor, M., Udry, S., \& Queloz, D. 2000b, A\&A, 355, 581

Han, I., Black, D. C., \& Gatewood, G. 2001, ApJ, 548, L57

Hatzes, A. P., Cochran, W. D., McArthur, B., et al. 2000, ApJ, 544, L145

Heintz, W. D. 1978, Double stars (D. Reidel Publishing Company)

Høg, E., Fabricius, C., Makarov, V. V., Urban, S., et al. 2000, A\&A, 355, L27

Korzennik, S. G., Brown, T. M., Fischer, D. A., Nisenson, P., \& Noyes, R. W. 2000, ApJ, 533, L147

Kürster, M., Endl, M., Els, S., et al. 2000, A\&A, 353, L33

LLoyd, J. P., Liu, M. C., Graham, J. R., et al. 2000, in Planetary Systems in the Universe: Observation, Formation and Evolution IAU Symposium 202, ASP
Conference Series \#, ed. A. J. Penny, P. Artymowicz, A. M. Lagrange, \& S. S. Russell

Marcy, G. W., \& Butler, R. P. 1996, ApJ, 464, L147

Marcy, G. W., Butler, R. P., \& Vogt, S. S. 2000, ApJ, 536, L43

Marcy, G. W., Butler, R. P., Vogt, S. S., Fischer, D., \& Liu, M. C. 1999, ApJ, 520, 239

Mayor, M., Naef, D., Queloz, D., et al. 2000, in Planetary Systems in the Universe: Observation, Formation and Evolution, IAU Symposium 202, ASP Conf. Ser. \#, ed. A. J. Penny, P. Artymowicz, A. M. Lagrange, \& S. S. Russell

Mayor, M., \& Queloz, D. 1995, Nature, 378, 355

Mazeh, T., Naef, D., Torres, G., et al. 2000, ApJ, 532, L55

Mazeh, T., Zucker, S., Dalla Torre, A., \& van Leeuwen, F. 1999, ApJ, 522, L149

Naef, D., Mayor, M., Pepe, F., et al. 2000, in Disks, Planetesimals and Planets ASP Conference Series \#, ed. F. Garzón, C. Eiroa, D. de Winter, \& T. J. Mahoney

Naef, D., Mayor, M., Pepe, F., et al. 2001, A\&A, submitted

Noyes, R. W., Contos, A. R., Korzennik, S. G., et al. 1999, in Precise stellar radial velocities, IAU Colloquium 170, ASP Conf. Ser. \#185, ed. J. B. Hearnshaw, \& C. D. Scarfe

Penny, A. J., Artymowicz, P., Lagrange, A. M., \& Russell, S. S., eds. 2000, Planetary Systems in the Universe: Observation, Formation and Evolution

Perryman, M. A. C., Lindegren, L., Arenou, F., et al. 1996, A\&A, 310, L21

Pourbaix, D. 2001, A\&A, 369, L22

Queloz, D., Mayor, M., Naef, D., et al. 2000a, in Planetary Systems in the Universe: Observation, Formation and Evolution, IAU Symposium 202, ASP Conf. Ser. \#, ed. A. J. Penny, P. Artymowicz, A. M. Lagrange, \& S. S. Russell

Queloz, D., Mayor, M., Weber, L., et al. 2000b, A\&A, 354, 99 Robichon, N., \& Arenou, F. 2000, A\&A, 355, 295

Sivan, J. P., Mayor, M., Naef, D., et al. 2000, in Planetary Systems in the Universe: Observation, Formation and Evolution, IAU Symposium 202, ASP Conf. Ser. \#, ed. A. J. Penny, P. Artymowicz, A. M. Lagrange, \& S. S. Russell

Söderhjelm, S. 1999, IBVS, 4816, 1

Udry, S., Mayor, M., Naef, D., et al. 2000a, A\&A, 356, 590

Udry, S., Mayor, M., \& Queloz, D. 2000b, in Planetary Systems in the Universe: Observation, Formation and Evolution, IAU Symposium 202, ASP Conf. Ser. \#, ed. A. J. Penny, P. Artymowicz, A. M. Lagrange, \& S. S. Russell

Vogt, S. S., Marcy, G. W., Butler, R. P., \& Apps, K. 2000, ApJ, 536, 902

Zucker, S., \& Mazeh, T. 2000, ApJ, 531, L67 